\title{
Spinal Cord Stimulation Frequency Influences the Hemodynamic Response in Patients with Disorders of Consciousness
}

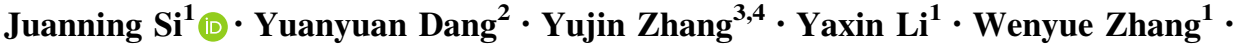 \\ Yi Yang ${ }^{2}$ Yue $\mathrm{Cui}^{3,4} \cdot$ Xiaoping Lou $^{1} \cdot$ Jianghong $\mathrm{He}^{2}{ }^{(\mathbb{D}} \cdot$ Tianzi Jiang $^{3,4,5,6,7}(\mathbb{D})$
}

Received: 24 January 2018/ Accepted: 27 May 2018/Published online: 11 July 2018

(C) The Author(s) 2018

\begin{abstract}
Spinal cord stimulation (SCS) is a promising technique for treating disorders of consciousness (DOCs). However, differences in the spatio-temporal responsiveness of the brain under varied SCS parameters remain unclear. In this pilot study, functional near-infrared spectroscopy was used to measure the hemodynamic responses of $10 \mathrm{DOC}$ patients to different SCS frequencies $(5 \mathrm{~Hz}, 10 \mathrm{~Hz}, 50 \mathrm{~Hz}, 70$ $\mathrm{Hz}$, and $100 \mathrm{~Hz}$ ). In the prefrontal cortex, a key area in consciousness circuits, we found significantly increased hemodynamic responses at $70 \mathrm{~Hz}$ and $100 \mathrm{~Hz}$, and significantly different hemodynamic responses between $50 \mathrm{~Hz}$ and
\end{abstract}

Jianghong $\mathrm{He}$

he_jianghong@sina.cn

Tianzi Jiang

jiangtz@nlpr.ia.ac.cn

1 School of Instrumentation Science and Opto-electronics Engineering, Beijing Information Science and Technology University, Beijing 100192, China

2 Department of Neurosurgery, People's Liberation Army General Hospital, Beijing 100700, China

3 Brainnetome Center, Institute of Automation, Chinese Academy of Sciences, Beijing 100190, China

4 National Laboratory of Pattern Recognition, Institute of Automation, Chinese Academy of Sciences, Beijing 100190, China

5 Key Laboratory for NeuroInformation of the Ministry of Education, School of Life Science and Technology, University of Electronic Science and Technology of China, Chengdu 625014, China

6 Chinese Academy of Sciences Center for Excellence in Brain Science, Institute of Automation, Chinese Academy of Sciences, Beijing 100190, China

7 Queensland Brain Institute, University of Queensland, St. Lucia, QL 4072, Australia
$70 \mathrm{~Hz} / 100 \mathrm{~Hz}$. In addition, the functional connectivity between prefrontal and occipital areas was significantly improved with SCS at $70 \mathrm{~Hz}$. These results demonstrated that SCS modulates the hemodynamic responses and long-range connectivity in a frequency-specific manner (with $70 \mathrm{~Hz}$ apparently better), perhaps by improving the cerebral blood volume and information transmission through the reticular formation-thalamus-cortex pathway.

Keywords Disorder of consciousness - Spinal cord stimulation · Frequency $\cdot$ Functional near-infrared spectroscopy $\cdot$ Hemodynamic response

\section{Introduction}

Advances in healthcare for brain injury have significantly increased the number of patients with chronic disorders of consciousness (DOCs) who survive brain injury [1]. However, there is no evidence-based treatment for DOC patients. The management of chronic DOCs is still challenging both for neuroscience and for clinical medicine [2].

Spinal cord stimulation (SCS) is a promising neuromodulation technique for the treatment of DOC patients. In SCS, electrodes implanted into the epidural space at $\mathrm{C} 2-\mathrm{C} 4$ deliver electrical impulses to stimulate the ascending reticular activating system and regulate the awareness circuit [3]. According to the literature, hypometabolism and impaired widespread network connectivity occur in brain circuits that are the neuronal substrates of consciousness in DOC patients [4-6]. It is well-established that the recovery of consciousness in DOC patients is correlated with the restoration of metabolism and the improvement of functional connectivity in the awareness circuits [4-6]. SCS has shown benefits in improving the cerebral blood perfusion and 
promoting the cortical neuroplasticity [2,7] of DOC patients, with better effects in clinical improvement than traditional pharmacological and non-pharmacological treatments [8-10], especially for minimally conscious state (MCS) patients [11, 12]. For instance, Yamamoto et al. reported that SCS increases the cerebral blood flow in MCS patients by $22.2 \%$ during the SCS period compared to baseline [11].

The parameters of SCS are programmable, such as frequency, amplitude, and pulse width [3]. The effects of SCS are markedly affected by these parameters. For example, frequency, which is a most important determinant of SCS, influences how often a neuron fires in response to an external stimulus [13]. Different frequencies of SCS therapy might have different neurochemical effects [13]. Therefore, the design of the frequency parameter is, theoretically, a key step in SCS treatment. However, in practice, the SCS frequency used in the clinical environment is determined primarily based on the subjective experience of clinicians and behavioral assessment scales [6, 14]. So far, studies concerning the effects of SCS frequency on DOCs are fragmented and limited. Among these limited studies, the frequency varies from 5 to $200 \mathrm{~Hz}$, among which some frequencies such as $70 \mathrm{~Hz}$ [10] and $100 \mathrm{~Hz}$ [15] have been found to improve the cerebral blood perfusion and produce acceptable behavioral enhancement in DOC patients after long-term clinical observation [2]. However, the differences in the spatio-temporal responsiveness at different SCS frequencies remain unknown, and it is unclear whether a frequency-specific effect exists. Therefore, there is a critical need to explore a rapid, quantitative method of evaluating differences in the spatio-temporal responsiveness of the brain at different SCS frequencies.

We have been investigating the influences of different SCS frequencies on the brain of DOC patients since the last two years, in order to clarify these issues. According to our previous EEG study, significantly altered relative power and synchronization in the frontal cortex of MCS patients occur after $5 \mathrm{~Hz}, 70 \mathrm{~Hz}$, or $100 \mathrm{~Hz}$ SCS [16]. However, owing to the interference of the electrical impulses of SCS with the EEG, the data were recorded before and immediately after the SCS procedure rather than during the SCS. Therefore, the real-time spatio-temporal responsiveness of the brain at different SCS frequencies remained uncertain. Functional near-infrared spectroscopy (fNIRS) [17-19] is a non-invasive, portable technology that is not subject to interference from electrical stimulation [18], and can be used for longitudinal monitoring, making it uniquely suited for investigating the real-time spatio-temporal responsiveness of brain activity in DOC patients during the SCS procedure. The feasibility of fNIRS-SCS measurement for DOC patients was demonstrated in our previous study [14]. In that study, we investigated the effects of the interval parameter of SCS and found that a shorter interval was better for improving the cerebral blood volume during the SCS procedure.

Therefore, in this pilot study, we used a portable fNIRS system to measure the real-time hemodynamic responses over the prefrontal and occipital areas during the SCS procedure and investigated the effects of SCS at different frequencies on the spatio-temporal responsiveness of DOC patients.

\section{Materials and Methods}

\section{Participants}

Ten DOC patients ( 5 males and 5 females, aged 17-64 years) were recruited from the Department of Neurosurgery, People's Liberation Army General Hospital, Beijing, China. Each patient was implanted with an SCS device about 1 month before the study and was in a stable clinical state. An SCS electrode (3587A, Medtronic Inc., Minneapolis, $\mathrm{MN}$ ) was implanted into the epidural space of the cervical vertebrae at the C2-C4 levels (Fig. 1). The corresponding implantable pulse generator was arranged subcutaneously on the collarbone. An SCS titration phase began 30 days after implantation. The clinical features of the DOC patients are listed in Table 1. The JFK Coma Recovery Scale was used to assess the state of consciousness of each DOC patient before SCS surgery [20]. Written informed consent was given by the patient's caregivers. This study was approved by the Ethics Committee of the PLA Army General Hospital.

\section{Study Design}

The experimental paradigm was block-designed (Fig. 2A). The stimulation parameters for SCS were configured by a wireless controller. Specifically, the SCS parameters were as follows: frequency $5 \mathrm{~Hz}, 10 \mathrm{~Hz}, 50 \mathrm{~Hz}, 70 \mathrm{~Hz}$, and 100 $\mathrm{Hz}$; pulse width $210 \mu \mathrm{s}$; duration $30 \mathrm{~s}$, and washout period 3 min, based on our previous study [14]. Because the neuronal and physiological sensitivity differed greatly among patients, we used different stimulation intensities (ranging from $1.0 \mathrm{~V}$ to $5.0 \mathrm{~V}$, without reaching the motor threshold) for different DOC patients, individually determined by an experienced clinician. Each patient received five different frequencies at a fixed stimulation intensity. Each session of SCS consisted of four blocks. To control the overall duration of the experiment and to avoid the influence of the previous session to the next session, each patient was given a 10-min rest after each session. The five frequencies were presented in a pseudo-random order. Each DOC patient received SCS on three different days with different sequences of frequencies. 
Fig. 1 X-ray images illustrating the location of implanted SCS devices. A An SCS electrode implanted in the epidural space at C2-C4. B An implantable impulse generator arranged subcutaneously on the collarbone.
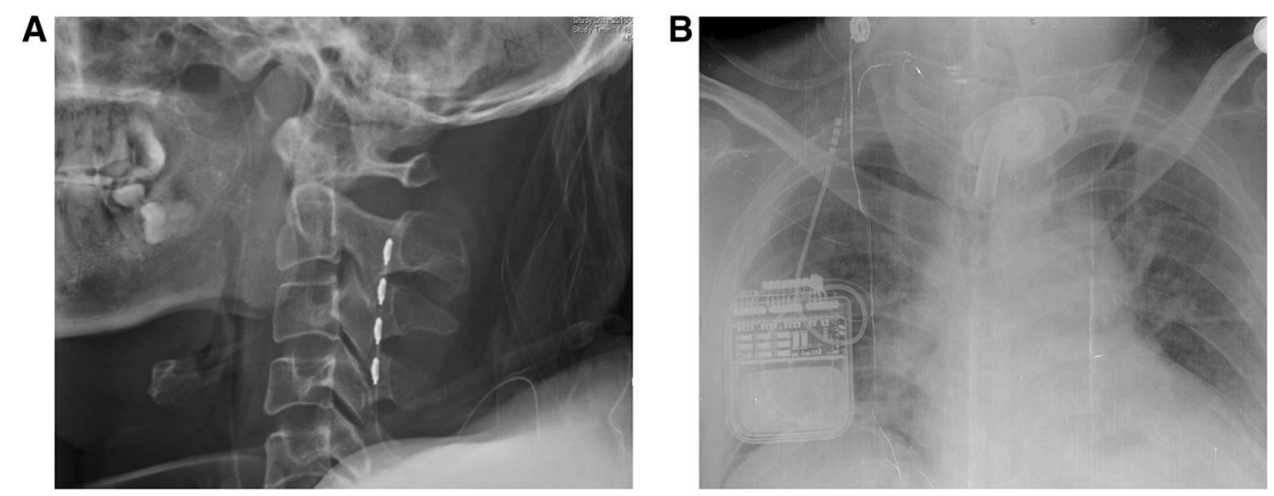

Table 1 Clinical features of patients with disorders of consciousness.

\begin{tabular}{llllrlc}
\hline No. & Diagnosis & Gender & Age (years) & Duration of DOC (months) & Etiology \\
\hline Patient 1 & VS & M & 17 & 4 & Head trauma \\
Patient 2 & VS & F & 17 & 11 & Head trauma \\
Patient 3 & VS & M & 18 & 8 & Anoxic \\
Patient 4 & MCS & F & 29 & 28 & Anoxic & 7 \\
Patient 5 & VS & F & 36 & 5 & Anoxic & Anoxic \\
Patient 6 & MCS & F & 41 & 3 & Stroke \\
Patient 7 & VS & M & 42 & 6 & Cerebral trauma \\
Patient 8 & VS & M & 53 & 12 & Cerebral hemorrhage \\
Patient 9 & VS & M & 54 & 11 & Cerebral hemorrhage \\
Patient 10 & VS & F & 64 & 24 & 7 \\
\hline
\end{tabular}

$V S$ vegetative state; $M C S$ minimally conscious state; $C R S$ - $R$ coma recovery scale-revised.

\section{fNIRS Recording}

The hemodynamic responses of the DOC patients were recorded using a custom-designed portable fNIRS system [21]. Two pads covered with optodes were placed on the scalp above the prefrontal and occipital areas. Each pad consisted of two sources and two detectors, yielding 4 optical channels (Fig. 2B). The distance between the source and detector pairs was $3 \mathrm{~cm}$, and each pad covered an area of $3 \times 3 \mathrm{~cm}^{2}$. The sampling rate of the fNIRS was $100 \mathrm{~Hz}$.

\section{Data Analysis}

Data were processed using the MatLab 2013a platform (MathWorks Inc., Natick, MA). First, the relative concentration changes of oxygenated, deoxygenated, and total $(\mathrm{HbT})$ hemoglobin were calculated based on the modified Beer-Lambert Law $[22,23]$. Since the HbT concentration is proportional to changes in regional cerebral blood volume [24, 25], we specified the hemodynamic responses mainly on the basis of the HbT concentration. Second, the hemodynamic data were low-pass filtered at $0.3 \mathrm{~Hz}$ to remove task-unrelated noise. Then the data were segmented into several epochs, starting $30 \mathrm{~s}$ before the SCS onset and ending $90 \mathrm{~s}$ after the stimulation, and epochs with large artifacts were rejected. After baseline correction (calculated from the 30-s epoch before SCS onset), the block-averaged hemodynamic responses were calculated. Next, the block-averaged hemodynamic responses were averaged across the channels over the prefrontal and occipital areas, separately. Finally, the group-averaged hemodynamic responses were calculated.

The functional connectivity was evaluated by calculating the correlation values of the time courses of the hemodynamic responses between the prefrontal and occipital areas for the five frequencies. First, the whole time courses of the hemodynamic responses were averaged across the four channels over the prefrontal and occipital areas, separately. Then, the time courses of the $80 \mathrm{~s}$ before the SCS onset were extracted as segmentations for further calculation. Next, the correlation values of the segmentations between the prefrontal and occipital areas were calculated. The first correlation value indicated the functional connectivity before the SCS procedure, whereas the averaged correlations for the remaining segmentations presented the functional connectivity after the SCS procedure.

All data are presented as mean \pm standard error unless otherwise stated. The $t$-test and one-way analysis of 
Fig. 2 The experimental configuration. A Experimental paradigm for SCS. B Arrangement of probes over the prefrontal and occipital areas. C Photograph of the experimental setup.
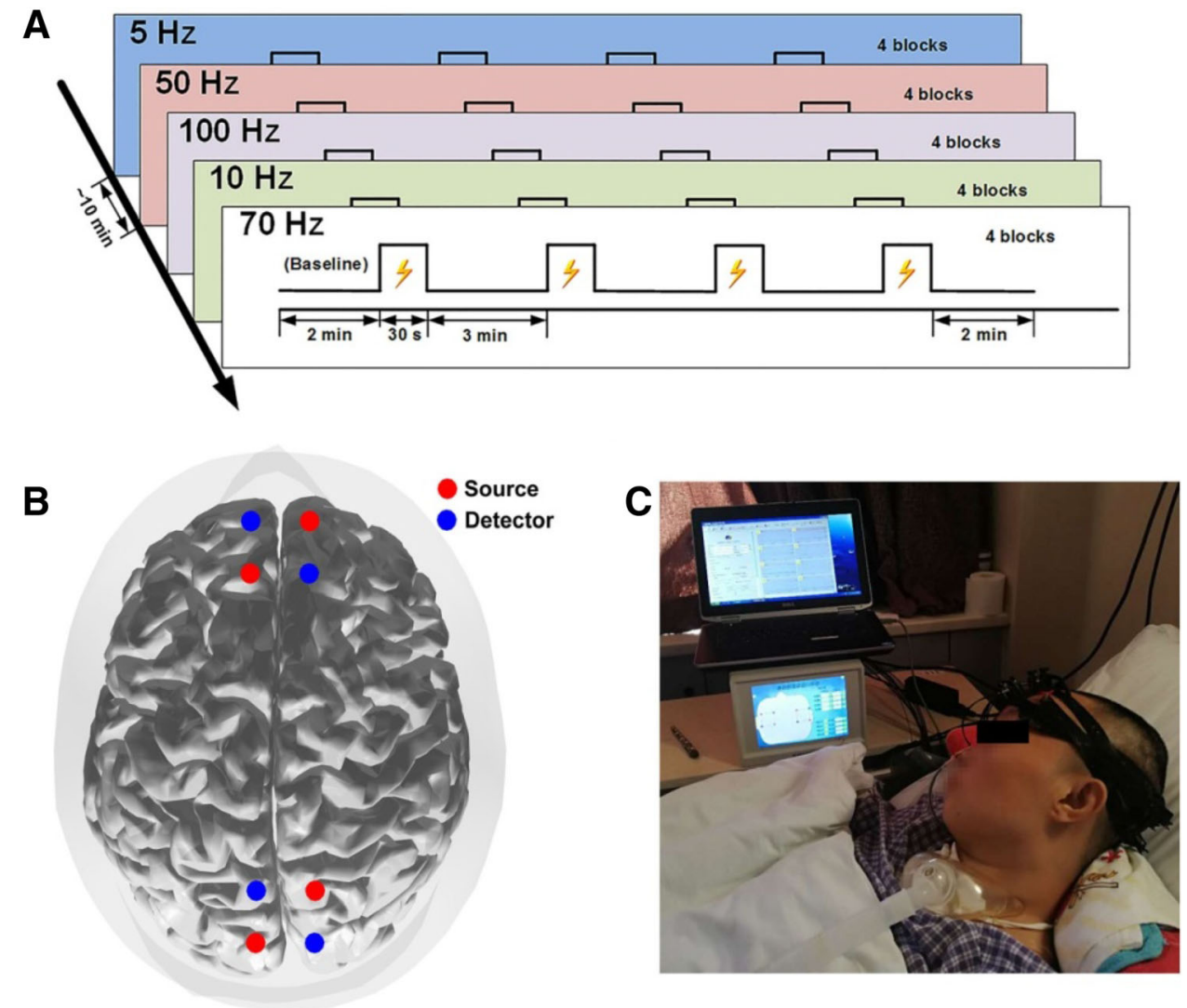

variance (ANOVA) were used to characterize the differences between different conditions. For post hoc analysis, the least-significant difference (LSD) algorithm was used. Differences were accepted as significant when $P<0.05$.

\section{Results}

During the baseline (pre-SCS) period, the HbT concentrations over the prefrontal and occipital areas at all five frequencies were relatively stable (Fig. 3). At SCS onset, the HbT concentrations over the prefrontal and occipital areas at different frequencies changed in different ways. Specifically, at the prefrontal area, the HbT concentration was significantly higher during the on-SCS period of the 70 $\mathrm{Hz}$ SCS at the group level in terms of positive peak $(P=$ $0.026)$ and mean $(P=0.039)$ values. However, there were no significant changes during the on-SCS period at the other SCS frequencies. After the SCS was turned off (postSCS), the mean HbT concentration at $100 \mathrm{~Hz}$ was significantly different from baseline at the group level $(P=0.031)$. As for the occipital area, there were no significant changes at any frequencies at the group level.

To further explore the differences between the SCSevoked hemodynamic fluctuations at the different frequencies, the peak and mean values of the hemodynamic responses during the on-SCS period were compared among the five frequencies using one-way ANOVA (Fig. 4). In the prefrontal area, although the peak $(F(4,45)=1.904, P=$ $0.126)$ and the mean $(F(4,45)=1.769, P=0.152)$ values did not significantly differ among the five frequencies, the values at $70 \mathrm{~Hz}$ were significantly higher than those at $50 \mathrm{~Hz}$ (peak value: $P=0.014$, LSD corrected; mean value: $P=0.028$, LSD corrected). In the occipital area, there were no significant differences in either the peak or the mean values between any frequencies at the group level.

In addition, the mean HbT values during the post-SCS period showed a trend of significant difference among the five frequencies $(F(4,45)=2.228, P=0.081)$ in the prefrontal area. Specifically, there were significant differences between $50 \mathrm{~Hz}$ and $70 \mathrm{~Hz}(P=0.039$, LSD corrected), and between $50 \mathrm{~Hz}$ and $100 \mathrm{~Hz}(P=0.015$, LSD corrected). This finding was consistent with that during the on-SCS period. As for the occipital cortex, there were no significant differences between any pair-wise conditions.

We further analyzed the short-term dynamic changes of the hemodynamic responses to stimulation at different frequencies, specifically by comparing the mean values of HbT concentrations in the pre-SCS, on-SCS, and post-SCS periods using one-way ANOVA (Fig. 5). At the prefrontal cortex, although there was no significant difference among the three periods with $70-\mathrm{Hz} \operatorname{SCS}(F(2,27)=1.855, P=$ 

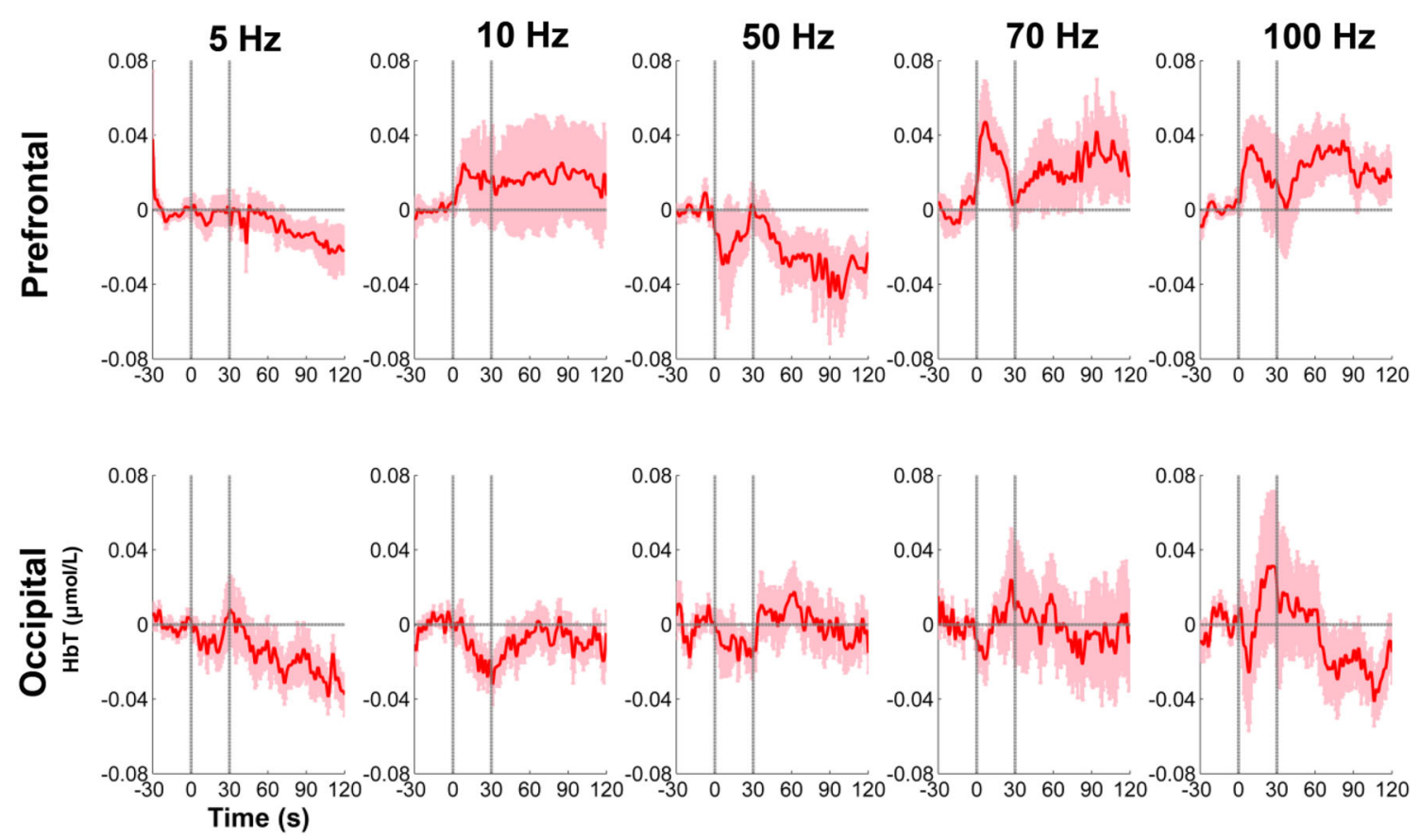

Fig. 3 Group-averaged hemodynamic responses of DOC patients during the SCS procedure in the prefrontal and occipital areas. The

lines. The shadowed areas indicate the standard errors of the mean SCS duration is indicated by the space between the two vertical gray

Fig. 4 Peak and mean values of the HbT concentrations among the five SCS frequencies during the on-SCS period. Error bars indicate the standard errors of the mean $(* P<0.05)$.
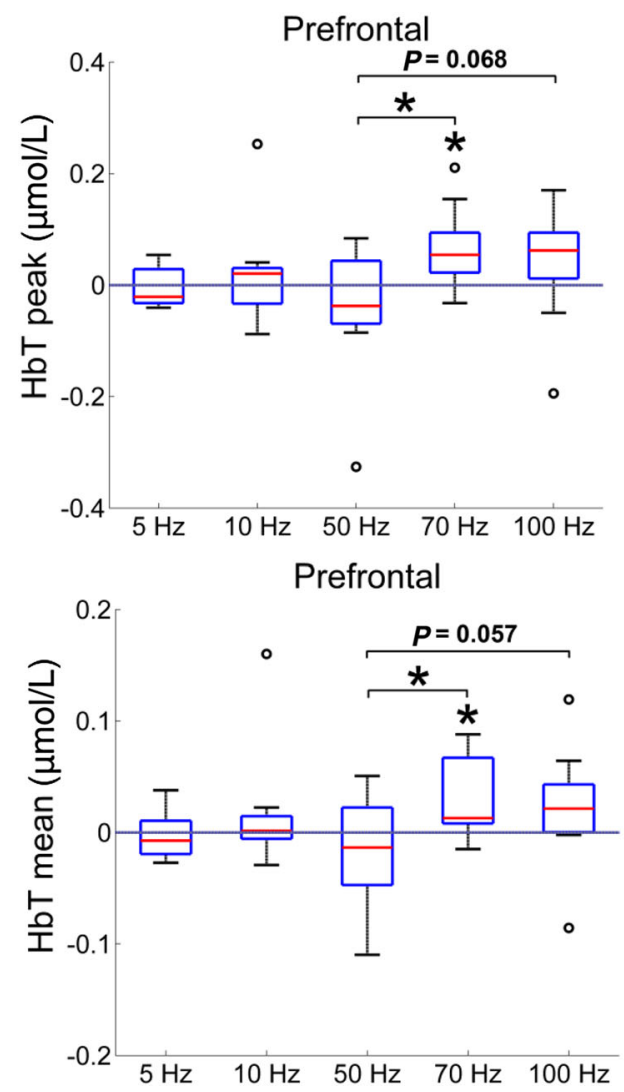

Occipital
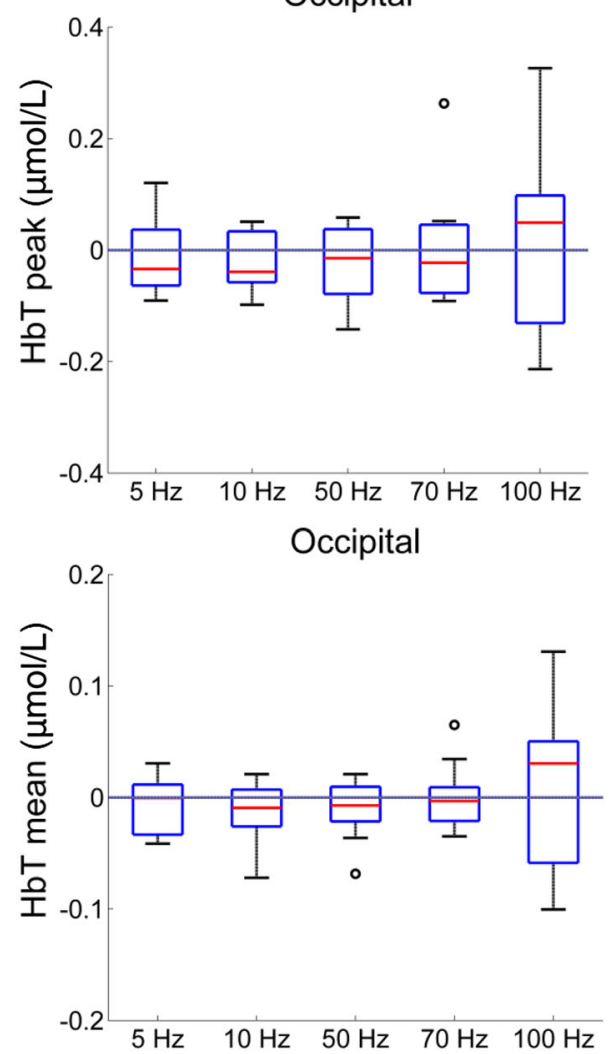
0.176), the difference between the on-SCS and pre-SCS periods still trended toward significant $(P=0.076$, LSD corrected). Similarly, at $100 \mathrm{~Hz} \mathrm{SCS}$, the difference between the post-SCS and pre-SCS periods trended toward significance $(P=0.081$, LSD corrected). At the other frequencies, pair-wise comparisons did not show any significant differences among the three periods.

To further explore the effects of SCS on the functional integrity of the brain, we calculated the functional connectivity using ROI-based correlation analysis between the prefrontal and occipital areas. At 5, 10, 50, and $70 \mathrm{~Hz}$, there were clear increases in functional connectivity after the SCS procedure compared to the period before SCS onset, but the increase was statistically significant only at $70 \mathrm{~Hz}(P=0.01)$ (Fig. 6). At the individual level, $70-\mathrm{Hz}$ SCS induced functional connectivity improvement in $80 \%$ $(8 / 10)$ of the DOC patients. By combining the hemodynamic responses and the functional connectivity, 50\% (4/8) of those with improved functional connectivity after $70-\mathrm{Hz}$ SCS also showed the largest increases of the hemodynamic response with 70-Hz SCS.

\section{Discussion}

The effective utility of SCS in the treatment for DOC patients necessitates an understanding of the effects of different SCS parameters on the brain. In this pilot study, we used fNIRS to measure the hemodynamic changes in real time over the prefrontal and occipital areas of DOC patients during the SCS procedure. The differences in cerebral hemodynamic responses and functional connectivity were evaluated to investigate the effects of SCS at different frequencies in DOC patients.

\section{Effects of Different SCS Frequencies on Hemody- namic Responses}

The distributions of the SCS-evoked hemodynamic responses of the five frequencies $(5 \mathrm{~Hz}, 10 \mathrm{~Hz}, 50 \mathrm{~Hz}, 70$ $\mathrm{Hz}$, and $100 \mathrm{~Hz}$ ) were clearly distinct, implying that the SCS frequency modulates the spatio-temporal responsiveness of brain activity in DOC patients. Specifically, at 70 $\mathrm{Hz}$, we found significant increases in the peak values, as well as the mean values, of the HbT concentration in the prefrontal cortex during the SCS procedure. Significant increases in the mean values of the hemodynamic responses were also found in the post-SCS period at 100 $\mathrm{Hz}$. This implies that SCS at $70 \mathrm{~Hz}$ and $100 \mathrm{~Hz}$ improves the cerebral blood volume and metabolism in DOC patients, and the SCS-evoked cerebral blood volume changes occur earlier at $70 \mathrm{~Hz}$ than at $100 \mathrm{~Hz}$. Interestingly, both the mean and the peak values of the SCSevoked hemodynamic responses during $50-\mathrm{Hz}$ stimulation in the prefrontal cortex were significantly (trended toward significantly) different from those at $70 \mathrm{~Hz}(100 \mathrm{~Hz})$,
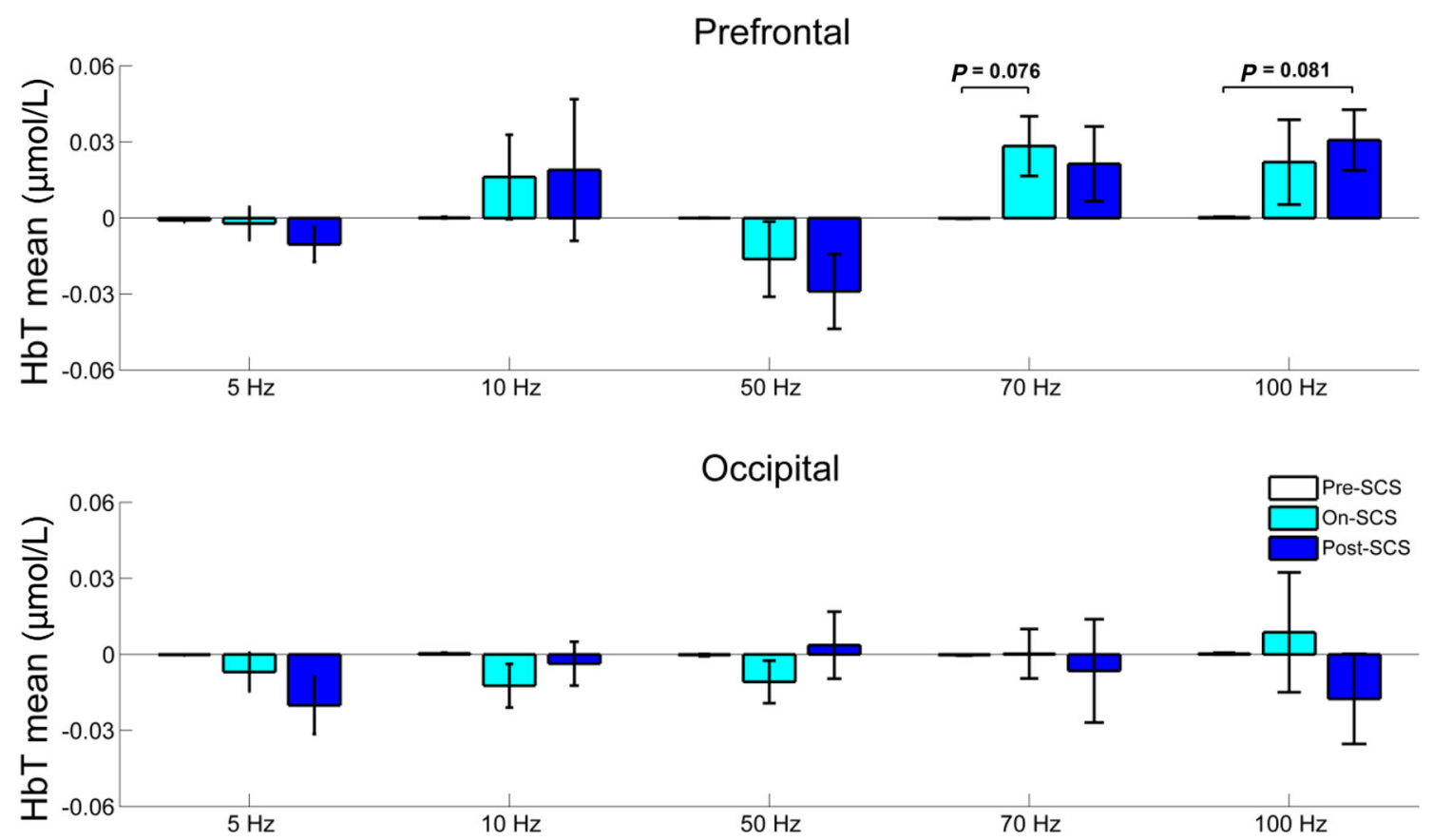

Fig. 5 Mean HbT concentrations at the five different SCS frequencies in the pre-, on-, and post-SCS periods. Error bars indicate the standard errors of the mean $(* P<0.05)$. 
Fig. 6 Effects of SCS on the functional integrity of the brain. A Group-level results of functional connectivity between the prefrontal and occipital areas for the five frequencies in the period before and after the SCS procedure. Red lines indicate positive, and blue lines negative correlation values. B Grouplevel functional connectivity between the periods before and after the SCS procedure for the five frequencies. White and black bars represent the means of the functional connectivity coefficients before and after the SCS procedure, respectively. Error bars indicate the standard error of the mean $(* P<0.05)$.

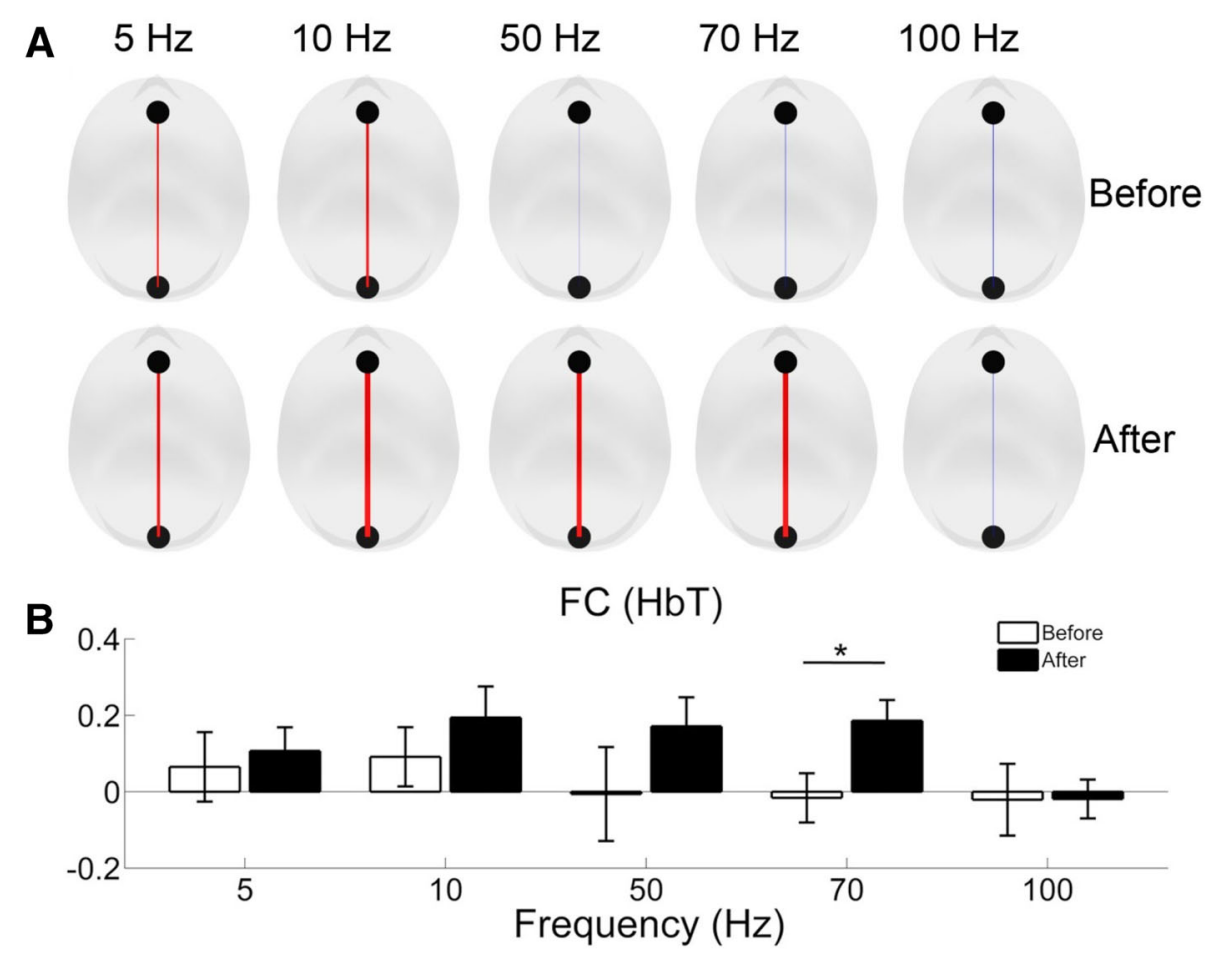

implying that the underlying effects of $50-\mathrm{Hz}$ SCS might differ from those at $70 \mathrm{~Hz}$ and $100 \mathrm{~Hz}$.

Above all, these findings showed that SCS modulates the hemodynamic responses of DOC patients in a frequency-specific manner. We suggest that the frequencyspecific effects of SCS might be due to the different neurochemical effects induced by SCS. For example, as reported by Miller et al., when applying SCS through implants in the dorsal spinal cord for the management of neuropathic pain, the stimulation frequency is a key determinant for activating different neurochemical mechanisms. Specifically, low frequencies $(2 \mathrm{~Hz}-10 \mathrm{~Hz})$ activate $\mu$-opioid receptor pathways, whereas high frequencies $(\sim 100 \mathrm{~Hz})$ activate endogenous $\delta$-opioid receptor pathways. Frequencies $\sim 50 \mathrm{~Hz}$ activate dorsal horn GABAergic neurons [26]. However, because of the different target areas in which the stimulator was implanted and the different pathological mechanisms underlying neuropathic pain and DOCs, whether such neurochemical effects of SCS on neuropathic pain also work on DOCs remains unknown and needs to be further explored.

\section{Effects of Different SCS Frequencies on Functional Connectivity}

It has been reported that DOCs might not only be associated with hypometabolism in widespread networks, but also with significantly decreased functional connectivity in the whole brain, especially long-distance functional connectivity [5, 27-29]. Interestingly, a study on DOC patients who regained consciousness showed functional restoration of metabolism and increased functional connectivity between the prefrontal cortex, thalamus, and other brain areas, and provided an important causal link between the functional integrity of the brain and consciousness [4, 29-32]. In our study, one kind of long-distance connectivity - functional connectivity between the prefrontal and occipital areas - significantly increased during $70-\mathrm{Hz}$ SCS at the group level. At the individual level, $70-\mathrm{Hz}$ SCS improved the functional connectivity for $80 \%(8 / 10)$ of DOC patients. Among them, $50 \%$ (4/8) also showed the largest increases of the hemodynamic response with $70-\mathrm{Hz}$ SCS. These findings provide insights that SCS not only modulates the cerebral blood volume of DOC patients in a frequency-specific manner, but also improves the between-network connectivity (at least that between the prefrontal and occipital areas) at a specific frequency.

\section{Possible Mechanism Underlying SCS for the Treat- ment of DOC Patients}

According to the mesocircuit model, in severe brain injuries, broad deafferentation and disconnection in the cortico-striatopallidal-thalamocortical loop results in a significant loss of input to the medium spiny neurons of 
the striatum, preventing these neurons from reaching their firing threshold [6]. Based on our findings, we suggest that a possible mechanism underlying SCS can be (at least partially) explained as follows: electrical modulation by SCS stimulates the ascending reticular formation-thalamuscortex pathway along with effects associated with improvements in cerebral blood volume and long-range functional connectivity of the brain circuits crucial for maintaining consciousness in the "mesocircuit" model $[6,33]$. The frequency-specific effects of SCS, especially the significant improvement of cerebral blood volume and long-distance functional connectivity at $70 \mathrm{~Hz}$, also explain the possible mechanism underlying good behavioral enhancement in DOC patients by $70-\mathrm{Hz}$ SCS $[2,10]$.

In addition, a significantly increased hemodynamic response in the prefrontal cortex and significantly improved prefrontal-occipital connectivity in DOC patients, indicate that the prefrontal cortex plays an important role in the effects of SCS on the cerebral cortex. Actually, it is wellestablished that the prefrontal cortex is one of the most important components underlying awareness [4, 6, 33]. First, studies in normal individuals examining conscious and nonconscious processing conditions have reported that prefrontal and parietal networks are associated with conscious access [34]. Second, transcranial magnetic stimulation over the prefrontal cortex can interrupt conscious perception and even disrupt conscious vision in normal individuals, also providing evidence that the prefrontal network plays a causal role in conscious perception $[19,35]$. Third, in pathological brain states, positron emission tomography studies have found hypometabolism and dysfunctional damage in the thalamus, prefrontal cortex, and frontoparietal association areas in DOC patients $[27,30,36]$. In the "mesocircuit" model, the frontal/prefrontal cortico-striatopallidal-thalamocortical loop system has been proposed to be crucial for maintaining consciousness [33]. In the current study, the $70-\mathrm{Hz}$ SCS-evoked hemodynamic responses in the prefrontal cortex were similar to those in typical tasks (such as working memory [37] and attention [38]). Moreover, based on a previous EEG study by our group, $70-\mathrm{Hz}$ SCS significantly alters the relative power and synchrony of the delta and gamma bands in the frontal area rather than other areas [16]. Therefore, we infer that the findings on the prefrontal cortex in this study may show its functional significance in the recovery of consciousness in DOC patients.

\section{Limitations of the Current Study}

Given that SCS is a newly-emerging neuromodulation technique for the treatment of DOC patients, the limited sample size and the wide patient-selection criteria are the main limitations of this pilot study. We hope that, with the development of neuromodulation techniques and the accumulation of patients among multiple centers, the current results will be confirmed and the specific effects of SCS parameters for DOC patients will be fully investigated. In addition, there is still a lack of long-term monitoring during routine SCS treatment or long-term clinical follow-up. Therefore, further investigation is needed on the relationship between the clinical effects and the frequency-specific effects on hemodynamic responses and functional connectivity. As the first study to investigate the hemodynamic and functional connectivity effects of SCS on the brains of DOC patients, it is clear that our current results are just the tip of the iceberg. We hope that our findings with significant frequency-specific effects provide some useful information for clinicians to design reasonable and effective SCS programming parameters for DOC patients. And since fNIRS is cost-effective, portable, and ecological and can be used for longitudinal monitoring, we hope it can be used to obtain new insights into brain function in this challenging patient population.

Acknowledgements This work was partially supported by the National Key Research and Development Program of China (2017YFB1002502), the National Natural Science Foundation of China (81501550, 81600919, and 31771076), the Cross Training (Shipei) Project of High-Caliber Talents in Beijing Municipal Institutions (2017-2018), the Supplementary and Supportive Project for Teachers at Beijing Information Science and Technology University (2018-2020, 5029011103), the School Scientific Research Project at Beijing Information Science and Technology University (1825010), the Beijing Municipal Science and Technology Commission (Z161100000516165), the Shenzhen Peacock Plan (KQTD2015033016104926), and the Guangdong Pearl River Talents Plan Innovative and Entrepreneurial Team grant (2016ZT06S220).

\section{Compliance with Ethical Standards}

Conflict of interest The authors declare that they have no conflict of interest.

Open Access This article is distributed under the terms of the Creative Commons Attribution 4.0 International License (http:// creativecommons.org/licenses/by/4.0/), which permits unrestricted use, distribution, and reproduction in any medium, provided you give appropriate credit to the original author(s) and the source, provide a link to the Creative Commons license, and indicate if changes were made.

\section{References}

1. Georgiopoulos M, Katsakiori P, Kefalopoulou Z, Ellul J, Chroni E, Constantoyannis C. Vegetative state and minimally conscious state: a review of the therapeutic interventions. Stereotact Funct Neurosurg 2010, 88: 199-207.

2. Della Pepa GM, Fukaya C, La Rocca G, Zhong J, Visocchi M. Neuromodulation of vegetative state through spinal cord stimulation: Where are we now and where are we going? Stereotact Funct Neurosurg 2013, 91: 275-287. 
3. Guerra A, Costantini EM, Maatta S, Ponzo D, Ferreri F. Disorders of consciousness and electrophysiological treatment strategies: a review of the literature and new perspectives. Curr Pharm Des 2014, 20: 4248-4267.

4. Laureys S, Schiff ND. Coma and consciousness: paradigms (re)framed by neuroimaging. Neuroimage 2012, 61: 478-491.

5. Di Perri C, Stender J, Laureys S, Gosseries O. Functional neuroanatomy of disorders of consciousness. Epilepsy Behav 2014, 30: 28-32.

6. Giacino JT, Fins JJ, Laureys S, Schiff ND. Disorders of consciousness after acquired brain injury: the state of the science. Nat Rev Neurol 2014, 10: 99-114.

7. Visocchi M, Della Pepa GM, Esposito G, Tufo T, Zhang W, Li S, et al. Spinal cord stimulation and cerebral hemodynamics: updated mechanism and therapeutic implications. Stereotact Funct Neurosurg 2011, 89: 263-274.

8. Funahashi K, Komai N, Ogura M, Kuwata T, Nakai M, Tsuji N. Effects and indications of spinal cord stimulation on the vegetative syndrome. No Shinkei Geka 1989, 17: 917-923.

9. Liu JT, Tan WC, Liao WJ. Effects of electrical cervical spinal cord stimulation on cerebral blood perfusion, cerebrospinal fluid catecholamine levels, and oxidative stress in comatose patients. Acta Neurochir Suppl 2008, 101: 71-76.

10. Kanno T, Morita I, Yamaguchi S, Yokoyama T, Kamei Y, Anil $\mathrm{SM}$, et al. Dorsal column stimulation in persistent vegetative state. Neuromodulation 2009, 12: 33-38.

11. Yamamoto T, Katayama Y, Obuchi T, Kobayashi K, Oshima H, Fukaya C. Spinal cord stimulation for treatment of patients in the minimally conscious state. Neurol Med Chir (Tokyo) 2012, 52: 475-481.

12. Yamamoto T, Katayama Y, Obuchi T, Kobayashi K, Oshima H, Fukaya C. Deep brain stimulation and spinal cord stimulation for vegetative state and minimally conscious state. World Neurosurg 2013, 80: S30.e31-39.

13. Schecklmann M, Dresler T, Beck S, Jay JT, Febres R, Haeusler J, et al. Reduced prefrontal oxygenation during object and spatial visual working memory in unpolar and bipolar depression. Psychiatry Res 2011, 194: 378-384.

14. Zhang Y, Yang Y, Si J, Xia X, He J, Jiang T. Influence of interstimulus interval of spinal cord stimulation in patients with disorders of consciousness: A preliminary functional near-infrared spectroscopy study. NeuroImage Clin 2018, 17: 1-9.

15. Fujii M, Sadamitsu D, Maekawa T, Uesugi S, Ozaki S, Koizumi $\mathrm{H}$, et al. Spinal cord stimulation therapy at an early stage for unresponsive patients with hypoxic encephalopathy. No Shinkei Geka 1998, 26: 315-321.

16. Bai Y, Xia X, Li X, Wang Y, Yang Y, Liu Y, et al. Spinal cord stimulation modulates frontal delta and gamma in patients of minimally consciousness state. Neuroscience 2017, 346: 247-254.

17. Jobsis FF. Noninvasive, infrared monitoring of cerebral and myocardial oxygen sufficiency and circulatory parameters. Science 1977, 198: 1264-1267.

18. Ferrari M, Quaresima V. A brief review on the history of human functional near-infrared spectroscopy (fNIRS) development and fields of application. Neuroimage 2012, 63: 921-935.

19. Yücel MA, Selb JJ, Huppert TJ, Franceschini MA, Boas DA. Functional near infrared spectroscopy: Enabling routine functional brain imaging. Curr Opin Biomed Eng 2017, 4: 78-86.

20. Schecklmann M, Romanos M, Bretscher F, Plichta MM, Warnke A, Fallgatter AJ. Prefrontal oxygenation during working memory in ADHD. J Psychiatr Res 2010, 44: 621-628.
21. Si J, Zhao R, Zhang Y, Zuo N, Zhang X, Jiang T. A portable fNIRS system with eight channels. Proc. SPIE 9305, Optical Techniques in Neurosurgery, Neurophotonics, and Optogenetics II, 93051B (10 March 2015). https://doi.org/10.1117/12.2080947.

22. Bozkurt A, Rosen A, Rosen H, Onaral B. A portable near infrared spectroscopy system for bedside monitoring of newborn brain. Biomed Eng Online 2005, 4: 29.

23. Kocsis L, Herman P, Eke A. The modified Beer-Lambert law revisited. Phys Med Biol 2006, 51: N91-N98.

24. Ferrari M, Wilson DA, Hanley DF, Traystman RJ. Effects of graded hypotension on cerebral blood flow, blood volume, and mean transit time in dogs. Am J Physiol 1992, 262: H1908H1914.

25. Yamashita Y, Maki A, Koizumi H. Wavelength dependence of the precision of noninvasive optical measurement of oxy-, deoxy-, and total-hemoglobin concentration. Med Phys 2001, 28: 1108-1114.

26. Miller JP, Eldabe S, Buchser E, Johanek LM, Guan Y, Linderoth B. Parameters of spinal cord stimulation and their role in electrical charge delivery: A review. Neuromodulation 2016, 19: 373-384.

27. Laureys S. The neural correlate of (un)awareness: lessons from the vegetative state. Trends Cogn Sci 2005, 9: 556-559.

28. Demertzi A, Soddu A, Laureys S. Consciousness supporting networks. Curr Opin Neurobiol 2013, 23: 239-244.

29. King JR, Sitt JD, Faugeras F, Rohaut B, El Karoui I, Cohen L, et al. Information sharing in the brain indexes consciousness in noncommunicative patients. Curr Biol 2013, 23: 1914-1919.

30. Laureys S, Goldman S, Phillips C, Van Bogaert P, Aerts J, Luxen A, et al. Impaired effective cortical connectivity in vegetative state: preliminary investigation using PET. Neuroimage 1999, 9: 377-382.

31. Chennu S, Annen J, Wannez S, Thibaut A, Chatelle C, Cassol H, et al. Brain networks predict metabolism, diagnosis and prognosis at the bedside in disorders of consciousness. Brain 2017, 140: 2120-2132.

32. Chennu S, Finoia P, Kamau E, Allanson J, Williams GB, Monti $\mathrm{MM}$, et al. Spectral signatures of reorganised brain networks in disorders of consciousness. PLoS Comput Biol 2014, 10: e1003887.

33. Schiff ND. Recovery of consciousness after brain injury: a mesocircuit hypothesis. Trends Neurosci 2010, 33: 1-9.

34. Taga G, Asakawa K, Maki A, Konishi Y, Koizumi H. Brain imaging in awake infants by near-infrared optical topography. Proc Natl Acad Sci U S A 2003, 100: 10722-10727.

35. Yasumura A, Inagaki M, Hiraki K. Relationship between neural activity and executive function: An NIRS study. ISRN Neurosci 2014, 2014: 734952.

36. Nakayama N, Okumura A, Shinoda J, Nakashima T, Iwama T. Relationship between regional cerebral metabolism and consciousness disturbance in traumatic diffuse brain injury without large focal lesions: an FDG-PET study with statistical parametric mapping analysis. J Neurol Neurosurg Psychiatry 2006, 77: 856-862.

37. Sato H, Yahata N, Funane T, Takizawa R, Katura T, Atsumori H, et al. A NIRS-fMRI investigation of prefrontal cortex activity during a working memory task. Neuroimage 2013, 83: 158-173.

38. Deepeshwar S, Vinchurkar SA, Visweswaraiah NK, Nagendra HR. Hemodynamic responses on prefrontal cortex related to meditation and attentional task. Front Syst Neurosci 2014, 8: 252. 